\title{
KATO SMOOTHING PROPERTIES OF A CLASS OF NONLINEAR DISPERSIVE WAVE EQUATIONS ON A PERIODIC DOMAIN*
}

\author{
Shu-Ming Sun ${ }^{1}$, Xin YAng ${ }^{2}$, Bing-Yu ZHANG ${ }^{3, *}$ And Ning ZhONG ${ }^{3}$
}

\begin{abstract}
The solutions of the Cauchy problem of the $\mathrm{KdV}$ equation on a periodic domain $\mathbb{T}$,

$$
u_{t}+u u_{x}+u_{x x x}=0, \quad u(x, 0)=\phi(x), \quad x \in \mathbb{T}, t \in \mathbb{R},
$$
\end{abstract}

possess neither the sharp Kato smoothing property,

$$
\phi \in H^{s}(\mathbb{T}) \Longrightarrow \partial_{x}^{s+1} u \in L_{x}^{\infty}\left(\mathbb{T}, L^{2}(0, T)\right),
$$

nor the Kato smoothing property,

$$
\phi \in H^{s}(\mathbb{T}) \Longrightarrow u \in L^{2}\left(0, T ; H^{s+1}(\mathbb{T})\right) .
$$

Considered in this article is the Cauchy problem of the following dispersive equations posed on the periodic domain $\mathbb{T}$,

$$
u_{t}+u u_{x}+u_{x x x}-g(x)(g(x) u)_{x x}=0, \quad u(x, 0)=\phi(x), \quad x \in \mathbb{T}, t>0,
$$

where $g \in C^{\infty}(\mathbb{T})$ is a real value function with the support

$$
\omega=\{x \in \mathbb{T}, g(x) \neq 0\}
$$

It is shown that

(1) if $\omega \neq \emptyset$, then the solutions of the Cauchy problem (1) possess the Kato smoothing property;

(2) if $g$ is a nonzero constant function, then the solutions of the Cauchy problem (1) possess the sharp Kato smoothing property.

Mathematics Subject Classification. 35B65, 35Q53, 35K45.

Received August 10, 2020. Accepted January 23, 2021.

\footnotetext{
* The paper is dedicated to Enrique Zuazua for his 60th birthday.

Keywords and phrases: Kato smoothing property, sharp Kato smoothing property, KdV equation, KdV-Burgers equation.

${ }^{1}$ Department of Mathematics, Virginia Polytechnic Institute and State University, Blacksburg, VA 24061, USA.

2 Department of Mathematics, University of California at Riverside, Riverside, CA 92521, USA.

3 Department of Mathematical Sciences, University of Cincinnati, Cincinnati, Ohio 45221, USA.

** Corresponding author: zhangb@ucmail.uc.edu
} 


\section{INTRODUCTION}

For the Cauchy problem of the $\mathrm{KdV}$ equation posed on $\mathbb{R}$,

$$
u_{t}+u u_{x}+u_{x x x}=0, \quad u(x, 0)=\phi(x), \quad x \in \mathbb{R}, t \in \mathbb{R}
$$

its solutions are as smooth as their initial values in the sense that

$$
u(\cdot, t) \in H^{s}(\mathbb{R}) \text { if and only if } \phi \in H^{s}(\mathbb{R}) \text { for any } t \in \mathbb{R} \text {. }
$$

However, Kato [17] discovered that the solutions of the KdV equation posed on $\mathbb{R}$ possess a local smoothing property:

$$
\phi \in H^{s}(\mathbb{R}) \Longrightarrow u \in L^{2}\left(-T, T ; H_{l o c}^{s+1}(\mathbb{R})\right) \text { for any } T>0
$$

This smoothing property, now referred to as the local Kato smoothing property, has been proved by Constantin and Saut [11] to be a common feature of dispersive-wave systems. Indeed, Constantin and Saut studied the following general dispersive-wave equation

$$
w_{t}+i P(D) w=0, \quad w(x, 0)=q(x), \quad x \in \mathbb{R}^{n}, t \in \mathbb{R},
$$

where $D=\frac{1}{i}\left(\partial / \partial x_{1}, \ldots, \partial / \partial x_{n}\right), P(D) w$ is the pseudo-differential operator

$$
P(D) w=\int_{\mathbb{R}^{n}} e^{i x \cdot \xi} p(\xi) \hat{w}(\xi) \mathrm{d} \xi
$$

defined by a real symbol $p(\xi)$, and $\hat{w}$ is the Fourier transformation of $w$ with respect to the spatial variable $x$. The symbol $p(\xi)$ is assumed to satisfy

(i) $p \in L_{l o c}^{\infty}\left(\mathbb{R}^{n}, \mathbb{R}\right)$ and is continuously differentiable for $|\xi|>R$ with some $R>0$,

(ii) there exist $m>1, C_{1}>0, C_{2}>0$ such that

$$
|p(\xi)| \leq C_{1}(1+|\xi|)^{m} \quad \text { for all } \quad \xi \in \mathbb{R}^{n}
$$

and

$$
\left|\partial p(\xi) / \partial \xi_{j}\right| \geq C_{2}(1+|\xi|)^{m-1}\left|\xi_{j}\right| /|\xi|
$$

for all $\xi \in \mathbb{R}^{n}$ with $|\xi|>R, j=1,2, \ldots, n$.

Theorem A (Constantin and Saut [11]) Let $s \geq-\frac{m-1}{2}$ be given. Then for any $q \in H^{s}\left(\mathbb{R}^{n}\right)$, the corresponding solution $w$ of (1.2) belongs to the space $C\left(\mathbb{R} ; H_{\text {loc }}^{s+\frac{m-1}{2}}\left(\mathbb{R}^{n}\right)\right)$ and moreover, for any given $T>0$ and $R>0$, there exists a constant $C$ depending only on $s, T$ and $R$ such that

$$
\int_{-T}^{T} \int_{|x| \leq R}\left|(I-\Delta)^{(m-1+2 s) / 4} w(x, t)\right|^{2} \mathrm{~d} x \mathrm{~d} t \leq C\|q\|_{H^{s}\left(\mathbb{R}^{n}\right)}^{2} .
$$

This local smoothing effect, as pointed out by Constantin and Saut in [11], is due to the dispersive nature of the linear part of the equation and the gain of the regularity, $(m-1) / 2$, depends only on the order $m$ of 
the equation and has nothing to do, in particular, with the dimension of the spatial domain $\mathbb{R}^{n}$. The discovery of the local Kato-smoothing property has stimulated enthusiasm in seeking various smoothing properties of dispersive-wave equations which, in turn, has greatly enhanced the study of mathematical theory of nonlinear dispersive-wave equations, in particular, the well-posedness of their Cauchy problems in low regularity spaces (see $[5,6,8,18-22]$ and see the references therein).

For the linear KdV equation (Airy equation)

$$
u_{t}+u_{x x x}=0, \quad u(x, 0)=\phi(x), \quad x \in \mathbb{R}, t \in \mathbb{R},
$$

the following estimate holds for its solutions.

Given $s \in \mathbb{R}$ and $R, T>0$, there exists a constant $C>0$ such that for any $\phi \in H^{s}(\mathbb{R})$, the corresponding solution $u$ of (1.4) satisfies

$$
\int_{-T}^{T} \int_{|x| \leq R}\left|\Lambda^{s+1} u(x, t)\right|^{2} \mathrm{~d} x \mathrm{~d} t \leq C\|\phi\|_{H^{s}(\mathbb{R})}^{2},
$$

where $\Lambda=\left(I-\partial_{x}^{2}\right)^{\frac{1}{2}}$.

Kenig et al. [19] have shown further that solutions of (1.4) possess the following stronger smoothing property.

Theorem B (Kenig et al. $[19,20])^{1}$ For any $s \in \mathbb{R}$, there exists a constant $C_{s}>0$ depending only on such that for any $\phi \in H^{s}(\mathbb{R})$, the corresponding solution $u$ of (1.4) satisfies

$$
\sup _{x \in \mathbb{R}}\left\|\partial_{x}^{s+1} u(x, \cdot)\right\|_{L_{t}^{2}(\mathbb{R})} \leq C_{s}\|\phi\|_{H^{s}(\mathbb{R})} .
$$

This property is now referred to as the sharp Kato-smoothing. ${ }^{2}$ It has become an important tool in studying the non-homogeneous boundary value problems $[1,2,13,14]$ and control theory of the KdV equation (see $[7,15,25,26]$ and the references therein).

Note that the local Kato smoothing and the sharp Kato smoothing properties as described by estimates (1.5) and (1.6) hold also for solutions of the linear KdV-Burgers (KdVB) equation posed on $\mathbb{R}$,

$$
u_{t}+u_{x x x}-\gamma u_{x x}=0, \quad u(x, 0)=\phi(x), \quad x \in \mathbb{R}, t \geq 0, \quad \gamma>0 .
$$

But, for the linear Burgers equation

$$
u_{t}-\gamma u_{x x}=0, \quad u(x, 0)=\phi(x), \quad x \in \mathbb{R}, t>0, \quad \gamma>0,
$$

while its solutions possess the (global) Kato smoothing property, i.e.,

for any $s \in \mathbb{R}$, there exists a constant $C_{s}>0$ depending only on $s$ such that for any $\phi \in H^{s}(\mathbb{R})$, the corresponding solution $u$ of (1.9) satisfies

$$
\int_{0}^{\infty} \int_{-\infty}^{\infty}\left|\Lambda^{s+1} u(x, t)\right|^{2} \mathrm{~d} x \mathrm{~d} t \leq C_{s}\|\phi\|_{H^{s}(\mathbb{R})}^{2}
$$

1 This smoothing property as described by (1.6) holds also for solutions of the nonlinear KdV equation (1.1) when $s>-\frac{3}{4}$.

2 The Kato smoothing property as described by (1.6) is also sharp in the sense that for any $\epsilon>0$, the estimate

$$
\int_{0}^{T} \int_{|x| \leq R}\left|\Lambda^{s+1+\epsilon} u(x, t)\right|^{2} \mathrm{~d} x \mathrm{~d} t \leq C\|\phi\|_{H^{s}(\mathbb{R})}^{2}
$$

fails to be true for the solutions of the Cauchy problem (1.4) with $C$ independent of $\phi$ (see [28]). 
it can only have the following weaker trace regularity estimate for its solutions.

For any $s \in \mathbb{R}$ and $\epsilon>0$, there exists a constant $C_{s, \epsilon}>0$ depending only on $s$ and $\epsilon$ such that for any $\phi \in H^{s}(\mathbb{R})$, the corresponding solution $u$ of (1.9) satisfies

$$
\sup _{x \in \mathbb{R}}\left\|\partial_{x}^{s+\frac{1}{2}-\epsilon} u(x, \cdot)\right\|_{L_{t}^{2}\left(\mathbb{R}^{+}\right)} \leq C_{s, \epsilon}\|\phi\|_{H^{s}(\mathbb{R})} .
$$

The local Kato 1 smoothing and the sharp Kato smoothing properties as described by (1.5) and (1.6) earlier are for solutions of the equations posed the whole line $\mathbb{R}$. For the Cauchy problem of the KdV equation posed on the periodic domain $\mathbb{T}$,

$$
u_{t}+u u_{x}+u_{x x x}=0, \quad u(x, 0)=\phi(x), \quad x \in \mathbb{T}, t \in \mathbb{R},
$$

however, in a sharp contrast, its solutions possess neither the sharp Kato smoothing property nor local the Kato smoothing property.

As for the Cauchy problem of the Burgers equation posed on $\mathbb{T}$,

$$
u_{t}+u_{x}+u u_{x}-\gamma u_{x x}=0, \quad u(x, 0)=\phi(x), \quad x \in \mathbb{T}, t>0, \quad \gamma>0,
$$

its solutions possess (global) Kato smoothing property

$$
\|u\|_{L^{2}\left(0, T ; H^{s+1}(\mathbb{T})\right)} \leq C_{s, T}\|\phi\|_{H^{s}(\mathbb{T})},
$$

but only possess the following trace regularity estimate

$$
\sup _{x \in \mathbb{T}}\left\|\partial_{x}^{s+\frac{1}{2}-\epsilon} u(x, \cdot)\right\|_{L^{2}(0, T)} \leq C_{s, \epsilon, T}\|\phi\|_{H^{s}(\mathbb{T})} .
$$

In particular, neither solutions of the $\mathrm{KdV}$ equation on $\mathbb{T}$ nor the solutions of the Burgers equation on $\mathbb{T}$ possess the sharp Kato smoothing property similar to that described by (1.6). It turns out that certain dissipation mechanism has to be introduced in order for solutions of the KdV equation on $\mathbb{T}$ to possess any Kato smoothing properties.

For solutions of the KdVB equation posed on $\mathbb{T}$,

$$
u_{t}+u u_{x}+u_{x x x}-\gamma u_{x x}=0, \quad u(x, 0)=\phi(x), \quad x \in \mathbb{T}, t>0, \quad \gamma>0,
$$

it is easy to see that they possess the (global) Kato smoothing property,

$$
\|u\|_{L^{2}\left(0, T ; H^{s+1}(\mathbb{T})\right)} \leq C_{s, T}\|\phi\|_{H^{s}(\mathbb{T})} .
$$

However, the question remains:

Do they possess the sharp Kato smoothing property,

$$
\sup _{x \in \mathbb{T}}\left\|\partial_{x}^{s+1} u(x, \cdot)\right\|_{L^{2}(0, T)} \leq C_{s, T}\|\phi\|_{H^{s}(\mathbb{T})} ?
$$

In this paper we will consider the Cauchy problem of the following class of the KdV equation posed on the 
TABLE 1. Results on $\mathbb{R}$.

\begin{tabular}{ccc}
\hline Smoothing Property & Kato & Sharp Kato \\
\hline KdV & Yes & Yes \\
Burgers & Yes & No \\
KdVB & Yes & Yes \\
\hline
\end{tabular}

TABLE 2. Results on $\mathbb{T}$.

\begin{tabular}{ccc}
\hline Smoothing Property & Kato & Sharp Kato \\
\hline KdV & No & No \\
Burgers & Yes & No \\
KdVB & Yes & $?$ \\
\hline
\end{tabular}

periodic domain $\mathbb{T}$ with certain dissipation embedded into the system:

$$
u_{t}+u u_{x}+u_{x x x}-g(x)(g(x) u)_{x x}=0, \quad u(x, 0)=u_{0}(x), \quad x \in \mathbb{T}, t>0
$$

where $g=g(x)$ is a $C^{\infty}$ smooth function on $\mathbb{T}$. Let

$$
\omega:=\{x \in \mathbb{T}, g(x) \neq 0\}
$$

For any smooth solution $u$ of (1.14), it holds that

$$
0=\frac{\mathrm{d}}{\mathrm{d} t} \int_{\mathbb{T}} u^{2}(x, t) \mathrm{d} x+2 \int_{\mathbb{T}}(g(x) u(x, t))_{x}^{2} \mathrm{~d} x=\frac{\mathrm{d}}{\mathrm{d} t} \int_{\mathbb{T}} u^{2}(x, t) \mathrm{d} x+2 \int_{\omega}(g(x) u(x, t))_{x}^{2} \mathrm{~d} x
$$

for any $t>0$. So the dissipation mechanism only acts on the set $\omega$, the part of the domain $\mathbb{T}$. We will show that as long as the set $\omega$ is not empty, no matter how small it is, the solution $u$ of (1.14) possesses (global) Kato smoothing property.

Theorem 1.1. (Kato smoothing property) Assume $g \in C^{\infty}(\mathbb{T})$ with nonempty support.

(i) Let $s \geq 0$ and $T>0$ be given. Then there exists a constant $C$ depending only on $s$ and $T$ such that any solution of the following linear problem,

$$
u_{t}+u_{x x x}-g(x)(g(x) u)_{x x}=f, \quad u(x, 0)=u_{0}(x), \quad x \in \mathbb{T}, t>0,
$$

satisfies

$$
\|u\|_{L^{\infty}\left(0, T ; H^{s}(\mathbb{T})\right)}+\|u\|_{L^{2}\left(0, T ; H^{s+1}(\mathbb{T})\right)} \leq C\left(\left\|u_{0}\right\|_{H^{s}(\mathbb{T})}+\|f\|_{L^{2}\left(0, T ; H^{s-1}(\mathbb{T})\right)}\right)
$$

for any $u_{0} \in H^{s}(\mathbb{T})$ and $f \in L^{2}\left(0, T ; H^{s-1}(\mathbb{T})\right)$.

(ii) Let $s \geq 0$ and $T>0$ be given. Then there exists a nondecreasing function $\alpha_{s, T}: \mathbb{R}^{+} \rightarrow \mathbb{R}^{+}$such that any solution of the Cauchy problem of the nonlinear equation (1.14) satisfies

$$
\|u\|_{L^{\infty}\left(0, T ; H^{s}(\mathbb{T})\right)}+\|u\|_{L^{2}\left(0, T ; H^{s+1}(\mathbb{T})\right)} \leq \alpha_{s, T}\left(\left\|u_{0}\right\|_{L^{2}(\mathbb{T})}\right)\left\|u_{0}\right\|_{H^{s}(\mathbb{T})}
$$


for any $u_{0} \in H^{s}(\mathbb{T})$. Moreover, if $s>\frac{1}{2}$, the solution $u$ can be written as

$$
u=v+w
$$

so that

$$
v \in C\left([0, T] ; H^{s}(\mathbb{T})\right) \cap L^{2}\left(0, T ; H^{s+1}(\mathbb{T})\right)
$$

solves the linear problem (1.15) with $f \equiv 0$ and

$$
w \in C\left([0, T] ; H^{s}(\mathbb{T})\right) \cap L^{2}\left(0, T ; H^{s+2}(\mathbb{T})\right)
$$

solves the linear problem (1.15) with

$$
f=-u u_{x}, \quad u_{0}(x)=0
$$

In the case of $\omega=\mathbb{T}$, there exists a $\gamma>0$ such that $g(x)>\sqrt{\gamma}$, or $g(x)<-\sqrt{\gamma}$, for any $x \in \mathbb{T}$. In this situation we assume that $g(x)=\sqrt{\gamma}$ is a constant function. The equation in (1.14) is then the KdV-Burgers equation. It will be shown that its solutions possess the sharp Kato smoothing property.

Theorem 1.2. (Sharp Kato smoothing property)

(i) Let $s \in \mathbb{R}$ be given. Then there exists a constant $C_{s}$ depending only on $s$ such that any solution $u$ of the Cauchy problem

$$
u_{t}+u_{x x x}-\gamma u_{x x}=0, \quad u(x, 0)=\phi(x), \quad x \in \mathbb{T}, t>0, \quad \gamma>0
$$

with $\phi \in H^{s}(\mathbb{T})$ satisfies

$$
\sup _{x \in \mathbb{T}}\left\|\partial_{x}^{s+1} u(x, \cdot)\right\|_{L^{2}\left(\mathbb{R}^{+}\right)} \leq C_{s}\|\phi\|_{H^{s}(\mathbb{T})}
$$

(ii) Let $s \geq 0$ and $T>0$ be given. There exists a nondecreasing function $\alpha_{s, T}: \mathbb{R}^{+} \rightarrow \mathbb{R}^{+}$such that any solution $u$ of the Cauchy problem

$$
u_{t}+u u_{x}+u_{x x x}-\gamma u_{x x}=0, \quad u(x, 0)=\phi(x), \quad x \in \mathbb{T}, t>0, \quad \gamma>0,
$$

with $\phi \in H^{s}(\mathbb{T})$ satisfies

$$
\sup _{x \in \mathbb{T}}\left\|\partial_{x}^{s+1} u(x, \cdot)\right\|_{L^{2}(0, T)} \leq \alpha_{s, T}\left(\|\phi\|_{H^{s}(\mathbb{T})}\right)
$$

For the linear problem (1.15), by the standard semigroup theory [24], it admits a unique solution $u \in$ $C\left(\left[0, T ; H^{s}(\mathbb{T})\right)\right.$ for any $u_{0} \in H^{s}(\mathbb{T})$ and $f \in L^{1}\left(0, T ; H^{s}(\mathbb{T})\right)$. Moreover, due to the role of the dissipative term $g(x)(g(x) u)_{x x}$,

$$
u \in L^{2}\left(0, T ; H^{s+1}(\omega)\right)
$$

and the solution $u$ is one order smoother than its initial value $\phi$ when viewed on $\omega$, a small open subset of $\mathbb{T}$. Our Theorem 1.1 shows not only on $\omega$, but also on the whole spatial domain $\mathbb{T}$, the solution $u$ is one order 
smoother than the regularity of the initial value $u_{0}$,

$$
u \in L^{2}\left(0, T ; H^{s+1}(\mathbb{T})\right) .
$$

This kind of phenomena is usually called as propagation of regularity in classical analysis: the $s+1$ regularity of $u$ has spreaded from the small open subset $\omega$ to the whole spatial domain $\mathbb{T}$. It will be proved using some standard techniques in analysis.

In the study of control and stabilization of distributed parameter systems described by some partial differential equations, it may happen that the systems lack certain smoothing properties which are needed to establish the controllability and stabilizability. One of the approaches to deal with this situation is to introduce some local dissipation mechanism through feedback control acting on a small part of the spatial domain and then show the gained local regularity can propagate to the whole spatial region so that the resulted closed loop systems possess the needed smoothing properties. The interested readers are referred to the recent works of Linares and Rosier [23] for control and stabilization of the Benjamin-Ono equation on a periodic domain, and Flores and Smith [12] for control and stabilization of the fifth order KdV equations on a periodic domain, as well as the references therein.

For the Cauchy problem of the linear KdV-Burgers equation posed on $\mathbb{R}$,

$$
u_{t}+u_{x x x}-\gamma u_{x x}=0, \quad u(x, 0)=\phi(x), \quad x \in \mathbb{R}, \quad t>0,
$$

its solution $u$ is given by

$$
u(x, t):=W_{\mathbb{R}}(t) \phi=\int_{\mathbb{R}} e^{i t \xi^{3}-\gamma \xi^{2} t} e^{i x \xi} \hat{\phi}(\xi) \mathrm{d} \xi=\int_{\mathbb{R}} e^{i t \eta-\gamma \mu^{2}(\eta) t} e^{i x \mu(\eta)} \mu^{\prime}(\eta) \hat{\phi}(\mu(\eta)) \mathrm{d} \eta
$$

Here, $\hat{\phi}(\xi)$ is the Fourier transform of $\phi(x)$, and $\mu(\eta)=\eta^{\frac{1}{3}}$ is the inverse function of $\eta=\xi^{3}$. Using Lemma 2.6 in [4] (a revised version of the Plancherel theorem) with respect to $t$ variable,

$$
\left\|u_{x}(x, \cdot)\right\|_{L_{t}^{2}\left(\mathbb{R}^{+}\right)}^{2} \leq C \int_{\mathbb{R}^{+}}\left|\mu(\eta) \mu^{\prime}(\eta) \hat{\phi}(\mu(\eta))\right|^{2} \mathrm{~d} \eta \leq C \int_{\mathbb{R}}|\hat{\phi}(\xi)|^{2} \mathrm{~d} \xi
$$

which gives us the sharp Kato smoothing of the solution $u$ :

$$
\sup _{x \in \mathbb{R}}\left\|u_{x}(x, \cdot)\right\|_{L_{t}^{2}\left(\mathbb{R}^{+}\right)} \leq C\|\phi\|_{L_{x}^{2}(\mathbb{R})} .
$$

However, this approach to establish the sharp Kato smoothing property of the linear KdV-Burgers equation on $\mathbb{R}$ fails completely for the equation posed on the periodic domain $\mathbb{T}$

$$
u_{t}+u_{x x x}-\gamma u_{x x}=0, \quad u(x, 0)=\phi(x), \quad x \in \mathbb{T}, \quad t>0,
$$

even though it has a similar explicit solution formula.

In this article, we propose a new approach to establish the sharp Kato smoothing property for solutions of the Cauchy problem (1.19). We study the following initial boundary value problem (IBVP for short) of the linear KdV-Burgers equation posed on the finite interval $(0,1)$

$$
\left\{\begin{array}{l}
u_{t}+u_{x x x}-\gamma u_{x x}=0, \quad u(x, 0)=\phi(x), \quad x \in(0,1), \\
u(0, t)-u(1, t)=0, \quad u_{x}(0, t)-u_{x}(1, t)=0, \quad u_{x x}(0, t)-u_{x x}(1, t)=0
\end{array}\right.
$$


which is equivalent to the Cauchy problem (1.19).

Consideration is first given to the associated non-homogeneous boundary value problem:

$$
\left\{\begin{array}{l}
v_{t}+v_{x x x}-\gamma v_{x x}=0, \quad v(x, 0)=0, \quad x \in(0,1), \\
v(0, t)-v(1, t)=h_{1}(t), \quad v_{x}(0, t)-v_{x}(1, t)=h_{2}(t), \quad v_{x x}(0, t)-v_{x x}(1, t)=h_{3}(t) .
\end{array}\right.
$$

For given $\vec{h}=\left(h_{1}, h_{2}, h_{3}\right)$, denote its solution by

$$
v(x, t)=\mathcal{B}(t) \vec{h}
$$

in which the operator $\mathcal{B}(t)$ is called the boundary integral operator. Formally, if $u$ is a solution of (1.18) and we let

$$
h_{1}(t)=u(0, t)-u(1, t), h_{2}(t)=u_{x}(0, t)-u_{x}(1, t), h_{3}(t)=u_{x x}(0, t)-u_{x x}(1, t),
$$

then

$$
w(x, t)=u(x, t)-\mathcal{B}(t) \vec{h}
$$

is a solution of $\operatorname{IBVP}(1.20)$ when restricted on the interval $(0,1)$. As the solution $u$ of $(1.18)$ (here $\phi(x)$ is zero outside of $(0,1))$ is known to possess the sharp Kato smoothing property

$$
\sup _{x \in(0,1)}\left\|u_{x}(x, \cdot)\right\|_{L^{2}(0, T)} \leq C\|\phi\|_{L^{2}((0,1))},
$$

one thus only needs to prove that $v=\mathcal{B}(t) \vec{h}$ satisfies

$$
\sup _{x \in(0,1)}\left\|v_{x}(x, \cdot)\right\|_{L^{2}(0, T)} \leq C\|\phi\|_{L^{2}(0,1)}
$$

in order to show that the solution of (1.20) possesses the sharp Kato smoothing property. Note that when $u$ is a solution of (1.18) with its initial value $\phi \in L^{2}(\mathbb{R})$, then

$$
g_{1}(t)=u(0, t)-u(1, t) \in H_{t}^{\frac{1}{3}}(0, T), \quad g_{2}(t)=u_{x}(0, t)-u_{x}(1, t) \in L_{t}^{2}(0, T),
$$

and

$$
g_{3}(t)=u_{x x}(0, t)-u_{x x}(1, t) \in H_{t}^{-\frac{1}{3}}(0, T)
$$

satisfying

$$
\left\|g_{1}\right\|_{H^{\frac{1}{3}}(0, T)}+\left\|g_{2}\right\|_{L^{2}(0, T)}+\left\|g_{3}\right\|_{H^{-\frac{1}{3}}(0, T)} \leq C\|\phi\|_{L^{2}(\mathbb{R})} .
$$

So the key for this strategy to work is to show that $v=\mathcal{B}(t) \vec{h}$ satisfies

$$
\|v\|_{C\left([0, T] ; L^{2}(0,1)\right)}+\left\|v_{x}\right\|_{L_{x}^{\infty}\left(0,1 ; L^{2}(0, T)\right)} \leq C\|\vec{h}\|_{\mathcal{H}(0, T)}
$$


for any

$$
\vec{h} \in \mathcal{H}(0, T):=H^{\frac{1}{3}}(0, T) \times L^{2}(0, T) \times H^{-\frac{1}{3}}(0, T) .
$$

It will be demonstrated later that the estimate (1.22) holds if and only if the dissipative parameter $\gamma>0$.

The paper is organized as follows. Section 2 is concerned with the Kato smoothing property of the system (1.15) and provides the proof of Theorem 1.1. Section 3 is devoted to establish the sharp Kato smoothing property of the KdV-Burgers equation following the strategy outlined earlier.

\section{KATO SMOOTHING PROPERTY}

In this section, we will present the proof of Theorem 1.1.

The usual $L^{2}(\mathbb{T})$ inner product is written $(u, v)=\int_{\mathbb{T}} u(x) v(x) \mathrm{d} x$, and in $H^{s}(\mathbb{T}), s \in \mathbb{R},(u, v)_{s}=((1-$ $\left.\left.\partial_{x}^{2}\right)^{\frac{s}{2}} u,\left(1-\partial_{x}^{2}\right)^{\frac{s}{2}} v\right)$. The norm in $H^{s}(\mathbb{T})$ is given by $\|u\|_{s}=(u, u)_{s}^{\frac{1}{2}}$, and we abbreviate $\|u\|_{0}=\|u\|$.

Consider first the linear problem

$$
u_{t}+u_{x x x}-g(x)(g(x) u)_{x x}=f, \quad u(x, 0)=u_{0}(x), \quad x \in \mathbb{T}, t>0 .
$$

Proposition 2.1. Let $s \geq 0, T>0$ be given. For any $u_{0} \in H^{s}(\mathbb{T}), f \in L^{2}\left(0, T ; H^{s-1}(\mathbb{T})\right)$, the Cauchy problem (2.1) admits a unique solution $u \in C\left(0, T ; H^{s}(\mathbb{T})\right) \cap L^{2}\left(0, T ; H^{s+1}(\mathbb{T})\right)$ which, moreover, satisfies

$$
\sup _{t \in(0, T)}\|u(\cdot, t)\|_{s}+\|u\|_{L^{2}\left(0, T ; H^{s+1}(\mathbb{T})\right)} \leq C\left(\left\|u_{0}\right\|_{s}+\|f\|_{L^{2}\left(0, T ; H^{s-1}(\mathbb{T})\right)}\right),
$$

where $C>0$ is a constant depending only on $s$ and $T$.

Proof. The constant $c$ in this proof may vary from line to line and only depends on $s, T$ and $g$.

By the standard semigroup theory, for any $u_{0} \in H^{s}(\mathbb{T})$ and $f \in L^{1}\left(0, T ; H^{s}(\mathbb{T})\right)$, the Cauchy problem $(2.1)$ admits a unique solution $u \in C\left([0, T] ; H^{s}(\mathbb{T})\right)$ and

$$
\sup _{t \in(0, T)}\|u(\cdot, t)\|_{s} \leq C_{s, T}\left(\left\|u_{0}\right\|_{s}+\|f\|_{L^{1}\left(0, T ; H^{s}(\mathbb{T})\right)}\right) .
$$

One thus only needs to show that estimate (2.2) holds for any smooth solution of (2.1). The proof is then completed by using the standard density arguments.

Consider first the case of $s=0$. Assume that $u$ is smooth solution of (2.1). Multiplying the both sides of the equation in $(2.1)$ by $2 u$ and integrating over $\mathbb{T}$ and $(0, t)$ lead to

$$
\|u(\cdot, t)\|^{2}+2 \int_{0}^{t}\left\|\partial_{x}(g u)\right\|^{2} \mathrm{~d} \tau \leq\left\|u_{0}\right\|^{2}+2 \int_{0}^{t}\|u\|_{1}\|f\|_{-1} \mathrm{~d} \tau
$$

for any $t \in(0, T]$. In particular,

$$
\sup _{0<t<T}\|u(\cdot, t)\|^{2} \leq\left\|u_{0}\right\|^{2}+2 \int_{0}^{T}\|u\|_{1}\|f\|_{-1} \mathrm{~d} \tau
$$

and

$$
\int_{0}^{T}\|u\|^{2} \mathrm{~d} t \leq T\left\|u_{0}\right\|^{2}+2 T \int_{0}^{T}\|u\|_{1}\|f\|_{-1} \mathrm{~d} \tau
$$


For $\omega=\{x \in \mathbb{T} ; g(x) \neq 0\}$, let $\omega_{x}=\{x+y, y \in \omega\}$ for given $x \in \omega$. As $\mathbb{T}$ is compact, there exist $x_{k} \in \mathbb{T}$ for $k=1,2, \ldots, N$ such that

$$
\mathbb{T}=\cup_{k=1}^{N} \omega_{x_{k}}
$$

and, moreover, there exist $\psi_{k}^{*} \in C_{0}^{\infty}\left(\omega_{k}\right), k=1,2,3, \ldots, N$, which forms a finite partition of unit,

$$
1=\sum_{k=1}^{N}\left(\psi_{k}^{*}(x)\right)^{2}, \quad \text { for any } x \in \mathbb{T} .
$$

For $k=1,2, \ldots, N$, define $\psi_{k}(x)=\psi_{k}^{*}\left(x+x_{k}\right)$. Then $\psi_{k} \in C_{0}^{\infty}(\omega)$,

$$
1=\sum_{k=1}^{N} \psi_{k}^{2}\left(x-x_{k}\right), \quad \text { for any } x \in \mathbb{T},
$$

and $\psi_{k}(x)=g(x) \tilde{\psi}_{k}(x)$ for some $\tilde{\psi}_{k} \in C^{\infty}(\mathbb{T})$. For each $k$, there exists a $\phi_{k} \in C^{\infty}(\mathbb{T})$ such that

$$
\psi_{k}^{2}(x)-\psi_{k}^{2}\left(x-x_{k}\right)=\phi_{k}^{\prime}(x)
$$

One has

$$
\begin{aligned}
\int_{0}^{T}\left\|u_{x}\right\|^{2} \mathrm{~d} t & =\sum_{k} \int_{0}^{T}\left(\psi_{k}^{2}\left(x-x_{k}\right) u_{x}, u_{x}\right) \mathrm{d} t \\
& \leq \sum_{k}\left|\int_{0}^{T}\left(\psi_{k}^{2}(x) u_{x}, u_{x}\right) \mathrm{d} t\right|+\sum_{k}\left|\int_{0}^{T}\left(\phi_{k}^{\prime}(x) u_{x}, u_{x}\right) \mathrm{d} t\right| \\
& :=\sum_{k} M_{1, k}+\sum_{k} M_{2, k},
\end{aligned}
$$

and

$$
\begin{aligned}
M_{1, k} & =\left|\int_{0}^{T}\left(\psi_{k}^{2}(x) u_{x}, u_{x}\right) \mathrm{d} t\right|=\int_{0}^{T}\left\|\tilde{\psi}_{k} g u_{x}\right\|^{2} \mathrm{~d} t \\
& \leq \int_{0}^{T}\left\|\tilde{\psi}_{k}(g u)_{x}\right\|^{2} \mathrm{~d} t+\int_{0}^{T}\left\|\tilde{\psi}_{k} g_{x} u\right\|^{2} \mathrm{~d} t \\
& \leq c \int_{0}^{T}\left\|\partial_{x}(g u)\right\|^{2} \mathrm{~d} t+c \int_{0}^{T}\|u\|^{2} \mathrm{~d} t .
\end{aligned}
$$

Thus, using (2.3) yields that

$$
M_{1, k} \leq c\left\|u_{0}\right\|^{2}+c \int_{0}^{T}\|u\|_{1}\|f\|_{-1} \mathrm{~d} t
$$

As for

$$
M_{2, k}=\left|\int_{0}^{T}\left(\phi_{k}^{\prime}(x) u_{x}, u_{x}\right) \mathrm{d} t\right|
$$


multiplying the both sides of the equation in (2.1) by $2 u \phi_{k}$ and integrating over $\mathbb{T}$, we arrive at

$$
\frac{\mathrm{d}}{\mathrm{d} t} \int u^{2} \phi_{k} \mathrm{~d} x+3 \int u_{x}^{2} \phi_{k}^{\prime} \mathrm{d} x+2 \int \partial_{x}\left(\phi_{k} g u\right) \partial_{x}(g u) \mathrm{d} x=\int u^{2} \phi_{k}^{(3)} \mathrm{d} x+\int u f \phi_{k} \mathrm{~d} x
$$

which yields that

$$
M_{2, k} \leq c\left\|u_{0}\right\|^{2}+c \int_{0}^{T}\|u\|^{2} \mathrm{~d} t+c \int_{0}^{T}\left\|\partial_{x}(g u)\right\|^{2} \mathrm{~d} t+c \int_{0}^{T}\|u\|_{1}\|f\|_{-1} \mathrm{~d} t
$$

Consequently,

$$
\int_{0}^{T}\|u\|_{1}^{2} \mathrm{~d} t \leq c\left\|u_{0}\right\|^{2}+c \int_{0}^{T}\|u\|_{1}\|f\|_{-1} \mathrm{~d} t \leq c\left\|u_{0}\right\|^{2}+\frac{1}{2} \int_{0}^{T}\|u\|_{1}^{2} \mathrm{~d} t+c \int\|f\|_{-1}^{2} \mathrm{~d} t
$$

and

$$
\int_{0}^{T}\|u\|_{1}^{2} \mathrm{~d} t \leq 2 c\left\|u_{0}\right\|^{2}+2 c \int_{0}^{T}\|f\|_{-1}^{2} \mathrm{~d} t
$$

Estimate (2.2) thus holds for $s=0$.

For $s=1$, let $v=u_{x}$, then $v$ solves

$$
v_{t}+v_{x x x}-g(x)(g(x) v)_{x x}-\Omega(u, v)=f_{x}, \quad v(x, 0)=v_{0}(x):=u_{0}^{\prime}(x), \quad x \in \mathbb{T}, t>0,
$$

with

$$
\Omega(u, v)=\Omega_{0} u+\Omega_{1} v+\Omega_{2} v_{x}
$$

and

$$
\begin{gathered}
\Omega_{0} u=g^{\prime}(x) g^{\prime \prime}(x) u+g(x) g^{\prime \prime \prime}(x) u \\
\Omega_{1} v=2 g^{\prime}(x) g^{\prime}(x) v+2 g(x) g^{\prime \prime}(x) v \\
\Omega_{2} v_{x}=2 g(x) g^{\prime}(x) v_{x}
\end{gathered}
$$

Arguing as earlier, one arrives at

$$
\|v\|_{L^{\infty}\left(0, T ; L^{2}(\mathbb{T})\right)}^{2}+2 \int_{0}^{T}\left\|\partial_{x}(g v)\right\|^{2} \mathrm{~d} \tau \leq 2\left\|v_{0}\right\|^{2}+\int_{0}^{T}\|v\|_{1}\left\|f_{x}\right\|_{-1} \mathrm{~d} \tau+2\left|\int_{0}^{T}(v, \Omega(u, v)) \mathrm{d} \tau\right| .
$$

It is easy to see that

$$
\left|\left(\Omega_{0} u, v\right)\right| \leq c\|u\|\|v\|, \quad\left|\left(\Omega_{1} v, v\right)\right| \leq c\|v\|^{2},
$$


and

$$
\left|\left(\Omega_{2} v_{x}, v\right)\right| \leq c\|v\|^{2} .
$$

Noticing that $v=u_{x}$ and invoking the estimate (2.2) with $s=0$, one arrives at

$$
2\left|\int_{0}^{T}(v, \Omega(u, v)) \mathrm{d} \tau\right| \leq c \int_{0}^{T}\left(\|u\|^{2}+\|v\|^{2}\right) \mathrm{d} t=c \int_{0}^{T}\|u\|_{1}^{2} \mathrm{~d} t \leq c\left(\left\|u_{0}\right\|^{2}+\|f\|_{L^{2}\left(0, T ; H^{-1}(\mathbb{T})\right)}^{2}\right)
$$

and

$$
\|v\|_{L^{\infty}\left(0, T ; L^{2}(\mathbb{T})\right)}^{2}+2 \int_{0}^{T}\left\|\partial_{x}(g v)\right\|^{2} \mathrm{~d} \tau \leq 2\left\|v_{0}\right\|^{2}+\int_{0}^{T}\|v\|_{1}\left\|f_{x}\right\|_{-1} \mathrm{~d} \tau+c\left(\left\|u_{0}\right\|^{2}+\|f\|_{L^{2}\left(0, T ; H^{-1}(\mathbb{T})\right)}^{2}\right) .
$$

The same propagation of regularity argument as in the case of $s=0$ yields

$$
\int_{0}^{T}\left\|v_{x}\right\|^{2} \mathrm{~d} \tau \leq c\left(\left\|v_{0}\right\|^{2}+\int_{0}^{T}\|v\|_{1}\left\|f_{x}\right\|_{-1} \mathrm{~d} \tau+\left\|u_{0}\right\|^{2}+\|f\|_{L^{2}\left(0, T ; H^{-1}(\mathbb{T})\right)}^{2}\right) .
$$

Consequently, arguing as in the case of $s=0$, it follows that the estimate (2.2) holds for $s=1$. The similar argument can show that the estimate (2.2) holds for any positive integer $s$. The case of $s$ being a positive fractional real number follows by interpolation.

Attention now is turned to the nonlinear problem

$$
u_{t}+u_{x x x}+u u_{x}-g(x)(g(x) u)_{x x}=0, \quad u(x, 0)=u_{0}(x), \quad x \in \mathbb{T}, t>0 .
$$

Proposition 2.2. Let $s \geq 0, T>0$ be given. For any $u_{0} \in H^{s}(\mathbb{T})$, (2.6) admits a unique solution $u \in$ $C\left(0, T ; H^{s}(\mathbb{T})\right) \cap L^{2}\left(0, T ; H^{s+1}(\mathbb{T})\right)$ which, moreover, satisfies

$$
\sup _{t \in(0, T)}\|u(\cdot, t)\|_{s}+\|u\|_{L^{2}\left(0, T ; H^{s+1}(\mathbb{T})\right)} \leq \alpha_{T}\left(\left\|u_{0}\right\|\right)\left\|u_{0}\right\|_{s}
$$

where $\alpha_{T}: \mathbb{R}^{+} \rightarrow \mathbb{R}^{+}$is a nondecreasing continuous function depending only on $T$.

Proof. For given $s \geq 0$ and $T>0$, let

$$
Y_{s, T}:=C\left([0, T] ; H^{s}(\mathbb{T})\right) \cap L^{2}\left(0, T ; H^{s+1}(\mathbb{T})\right) .
$$

First we show that (2.6) is locally well-posed in the space $H^{s}(\mathbb{T})$.

For a given $u_{0} \in H^{s}(\mathbb{T})$, we define a map $\Gamma$ on the space $Y_{s, T}$ : for any $v \in Y_{s, T}$,

$$
\Gamma(v):=u
$$

with $u$ as the solution of

$$
u_{t}+u_{x x x}-g(x)(g(x) u)_{x x}=-v v_{x}, \quad u(x, 0)=u_{0}(x), \quad x \in \mathbb{T}, t>0 .
$$


By the same arguments as those in the proof of Lemma 3.1 in [2], for any $v, w \in Y_{s, T}$,

$$
\int_{0}^{T}\left\|v w_{x}\right\|_{s} \mathrm{~d} \tau \leq C\left(T^{\frac{1}{3}}+T^{\frac{1}{2}}\right)\|v\|_{Y_{s, T}}\|w\|_{Y_{s, T}}
$$

Let $r>0$ and $\theta>0$ be constants to be determined, and

$$
S_{r, \theta}=\left\{v \in Y_{s, \theta}:\|v\|_{Y_{s, \theta}} \leq r\right\}
$$

For any $v \in S_{r, \theta}$, by Proposition 2.1

$$
\|\Gamma(v)\|_{Y_{s, \theta}} \leq c_{0}\left\|u_{0}\right\|_{s}+c_{1} \int_{0}^{\theta}\left\|v v_{x}\right\|_{s} \mathrm{~d} \tau \leq c_{0}\left\|u_{0}\right\|_{s}+c_{1}\left(\theta^{\frac{1}{2}}+\theta^{\frac{1}{3}}\right)\|v\|_{Y_{s, \theta}}^{2} .
$$

Choosing $r, \theta$ satisfying

$$
r=2 c_{0}\left\|u_{0}\right\|_{s}, \quad c_{1}\left(\theta^{\frac{1}{2}}+\theta^{\frac{1}{3}}\right) r \leq \frac{1}{2},
$$

then

$$
\|\Gamma(v)\|_{Y_{s, \theta}} \leq r
$$

for any $v \in S_{r . \theta}$. Thus, with such a choice of $r$ and $\theta, \Gamma$ maps $S_{r, \theta}$ into $S_{r, \theta}$. The same inequalities allow one to deduce that for $r$ and $\theta$ chosen as in (2.8),

$$
\left\|\Gamma\left(v_{1}\right)-\Gamma\left(v_{2}\right)\right\|_{Y_{s, \theta}} \leq \frac{1}{2}\left\|v_{1}-v_{2}\right\|_{Y_{s, \theta}}
$$

for any $v_{1}, v_{2} \in S_{r, \theta}$. In other words, $\Gamma$ is a contraction mapping of $S_{r, \theta}$, Its fixed point $u$ is the unique solution of (2.6) in $S_{r, \theta}$. More precisely, for any $u_{0} \in H^{s}(\mathbb{T})$, there exists a $\theta>0$ depending only $\left\|u_{0}\right\|_{s}$ such that $(2.6)$ admits a unique solution $u \in Y_{s, \theta}$.

Next we show that (2.6) is globally well-posed for which it suffices to prove that a global a-priori estimate

$$
\sup _{t \in(0, T)}\|u(\cdot, t)\|_{s} \leq \alpha_{T}\left(\left\|u_{0}\right\|\right)\left\|u_{0}\right\|_{s}
$$

holds. We only consider the case of $0 \leq s \leq 1$ and the proof for other values of $s$ is similar.

For $s=0$, as it follows from the equation in (2.6) that

$$
\frac{\mathrm{d}}{\mathrm{d} t} \int_{\mathbb{T}} u^{2}(x, t) \mathrm{d} x+2 \int_{\mathbb{T}}\left|\partial_{x}(g(x) u(x, t))\right|^{2} \mathrm{~d} x=0
$$

for any $t \geq 0$, we have

$$
\sup _{t \geq 0}\|u(\cdot, t)\| \leq\left\|u_{0}\right\| .
$$

Thus the solution $u$ exists globally, $u \in Y_{0, T}$ and

$$
\|u\|_{Y_{0, T}} \leq \alpha_{T}\left(\left\|u_{0}\right\|\right)\left\|u_{0}\right\| .
$$


For $s=1$, let $w=u_{x}$. Then, $w$ solves

$$
w_{t}+w_{x x x}+(u w)_{x}-g(g w)_{x x}-2\left(g g^{\prime \prime}+\left(g^{\prime}\right)^{2}\right) w-2 g^{\prime} g w_{x}=f, \quad w(x, 0)=u_{0}^{\prime}(x)
$$

This is a linear equation with variable coefficient $u \in Y_{0, T}$ and forcing

$$
f=\left(g^{\prime} g^{\prime \prime}+g g^{\prime \prime \prime}\right) u \text {. }
$$

Using the contraction mapping principle as before yields that for $u_{0} \in H^{1}(\mathbb{T}),(2.11)$ admits a unique solution $w \in Y_{0, T}$ satisfying

$$
\|w\|_{Y_{0, T}} \leq C\left\|u_{0}\right\|_{1} .
$$

Thus

$$
\left\|u_{x}\right\|_{Y_{0, T}} \leq C\left\|u_{0}\right\|_{1}
$$

where the constant $C$ depends only on $\|u\|_{Y_{0, T}}$ and therefore only on $\left\|u_{0}\right\|$ by (2.10). Consequently

$$
\sup _{0 \leq t \leq T}\|u(\cdot, t)\|_{1} \leq \alpha_{T}\left(\left\|u_{0}\right\|\right)\left\|u_{0}\right\|_{1}
$$

and moreover

$$
\sup _{0 \leq t \leq T}\|u(\cdot, t)\|_{s} \leq \alpha_{T}\left(\left\|u_{0}\right\|\right)\left\|u_{0}\right\|_{s}
$$

holds for $0<s<1$ by applying nonlinear interpolation [29].

Corollary 2.3. Let $s>\frac{1}{2}$. For any $u_{0} \in H^{s}(\mathbb{T})$, the corresponding solution $u$ of (2.6) can be decomposed into

$$
w=v+w
$$

with $v \in Y_{s, T}$ as the solution of (2.1) with $f \equiv 0$ satisfying

$$
\|v\|_{L^{2}\left(0, T ; H^{s+1}(\mathbb{T})\right)} \leq C\left\|u_{0}\right\|_{s},
$$

and $w \in C\left([0, T] ; H^{s}(\mathbb{T})\right) \cap L^{2}\left(0, T ; H^{s+2}(\mathbb{T})\right)$ as the solution of (2.1) with $u_{0} \equiv 0$ and $f=-u u_{x}$ satisfying

$$
\|w\|_{L^{2}\left(0, T ; H^{s+2}(\mathbb{T})\right)} \leq \alpha_{T}\left(\left\|u_{0}\right\|\right)\left\|u_{0}\right\|_{s},
$$

Proof. When $u_{0} \in H^{s}(\mathbb{T})$, the solution $u \in C\left([0, T] ; H^{s}(\mathbb{T})\right)$ with $u_{x} \in L^{2}\left(0, T ; H^{s}(\mathbb{T})\right)$. Thus, $u u_{x} \in$ $L^{2}\left(0, T ; H^{s}(\mathbb{T})\right)$ if $s>\frac{1}{2}$. The corollary follows from Proposition 2.1 .

\section{Sharp Kato SMOOThing PROPERTY}

In this section we present the proof of Theorem 1.2. Attentions are focused on the KdV-Burgers equation posed on the periodic domain $\mathbb{T}$,

$$
u_{t}+u u_{x}+u_{x x x}-\gamma u_{x x}=0, \quad u(x, 0)=u_{0}(x), \quad x \in \mathbb{T}, t>0 .
$$


The sharp Kato smoothing property will be established following the strategy outlined in Introduction. For simplicity we assume $\gamma=1$.

\subsection{Boundary integral operators}

Consideration is given to the following non-homogeneous boundary value problem for linear KdV-Burgers equation:

$$
\left\{\begin{array}{l}
u_{t}+u_{x x x}-u_{x x}=0, \quad u(x, 0)=0, \quad x \in(0,1) \\
u(0, t)-u(1, t)=h_{1}(t) \\
u_{x}(0, t)-u_{x}(1, t)=h_{2}(t) \\
u_{x x}(0, t)-u_{x x}(1, t)=h_{3}(t)
\end{array}\right.
$$

Applying the Laplace transform with respect to the temporal variable $t$ leads to

$$
\left\{\begin{array}{l}
s \tilde{u}+\tilde{u}_{x x x}-\tilde{u}_{x x}=0, \quad x \in(0,1), \\
\tilde{u}(0, s)-\tilde{u}(1, s)=\tilde{h}_{1}(s) \\
\tilde{u}_{x}(0, s)-\tilde{u}_{x}(1, s)=\tilde{h}_{2}(s) \\
\tilde{u}_{x x}(0, s)-\tilde{u}_{x x}(1, s)=\tilde{h}_{3}(s) .
\end{array}\right.
$$

where

$$
\tilde{f}(s)=\int_{0}^{\infty} e^{-s t} f(t) \mathrm{d} t, \text { with } \operatorname{Re} s>0
$$

For fixed $s=\alpha+i \beta$ with $\alpha>0$ and $s \neq \frac{4}{27}$, the equation

$$
s+\lambda^{3}-\lambda^{2}=0
$$

has three different solutions,

$$
\lambda_{j}=\lambda_{j}(s), j=1,2,3 .
$$

The solution $\tilde{u}$ of the equation (3.2) can be written as

$$
\tilde{u}(x, s)=\sum_{k=1}^{3} c_{k} e^{\lambda_{k}(s) x}
$$

where $c_{k}, k=1,2,3$ solve

$$
\left\{\begin{array}{l}
\left(1-e^{\lambda_{1}}\right) c_{1}+\left(1-e^{\lambda_{2}}\right) c_{2}+\left(1-e^{\lambda_{3}}\right) c_{3}=\tilde{h}_{1}, \\
\lambda_{1}\left(1-e^{\lambda_{1}}\right) c_{1}+\lambda_{2}\left(1-e^{\lambda_{2}}\right) c_{2}+\lambda_{3}\left(1-e^{\lambda_{3}}\right) c_{3}=\tilde{h}_{2}, \\
\lambda_{1}^{2}\left(1-e^{\lambda_{1}}\right) c_{1}+\lambda_{2}^{2}\left(1-e^{\lambda_{2}}\right) c_{2}+\lambda_{3}^{2}\left(1-e^{\lambda_{3}}\right) c_{3}=\tilde{h}_{3} .
\end{array}\right.
$$

Let

$$
\begin{aligned}
\Delta(s) & =\left|\begin{array}{ccc}
1-e^{\lambda_{1}} & 1-e^{\lambda_{2}} & 1-e^{\lambda_{3}} \\
\lambda_{1}\left(1-e^{\lambda_{1}}\right. & \lambda_{2}\left(1-e^{\lambda_{2}}\right) & \lambda_{3}\left(1-e^{\lambda_{3}}\right) \\
\lambda_{1}^{2}\left(1-e^{\lambda_{1}}\right) & \lambda_{2}^{2}\left(1-e^{\lambda_{2}}\right) & \lambda_{3}^{2}\left(1-e^{\lambda_{3}}\right)
\end{array}\right| \\
& =\left(1-e^{\lambda_{1}}\right)\left(1-e^{\lambda_{2}}\right)\left(1-e^{\lambda_{3}}\right)\left(\lambda_{3}-\lambda_{2}\right)\left(\lambda_{2}-\lambda_{1}\right)\left(\lambda_{3}-\lambda_{1}\right),
\end{aligned}
$$




$$
\begin{aligned}
& \Delta_{1}(s)=\left|\begin{array}{ccc}
\tilde{h}_{1} & 1-e^{\lambda_{2}} & 1-e^{\lambda_{3}} \\
\tilde{h}_{2} \lambda_{2}\left(1-e^{\lambda_{2}}\right) & \lambda_{3}\left(1-e^{\lambda_{3}}\right) \\
\tilde{h}_{3} & \lambda_{2}^{2}\left(1-e^{\lambda_{2}}\right) & \lambda_{3}^{2}\left(1-e^{\lambda_{3}}\right)
\end{array}\right| \\
& =\left(1-e^{\lambda_{2}}\right)\left(1-e^{\lambda_{3}}\right)\left[\tilde{h}_{1} \lambda_{2} \lambda_{3}\left(\lambda_{3}-\lambda_{2}\right)-\tilde{h}_{2}\left(\lambda_{3}-\lambda_{2}\right)\left(\lambda_{3}+\lambda_{2}\right)+\tilde{h}_{3}\left(\lambda_{3}-\lambda_{2}\right)\right], \\
& \Delta_{2}(s)=\left|\begin{array}{ccc}
1-e^{\lambda_{1}} & \tilde{h}_{1} & 1-e^{\lambda_{3}} \\
\lambda_{1}\left(1-e^{\lambda_{1}}\right) & \tilde{h}_{2} & \lambda_{3}\left(1-e^{\lambda_{3}}\right) \\
\lambda_{1}^{2}\left(1-e^{\lambda_{1}}\right) & \tilde{h}_{3} & \lambda_{3}^{2}\left(1-e^{\lambda_{3}}\right)
\end{array}\right| \\
& =\left(1-e^{\lambda_{1}}\right)\left(1-e^{\lambda_{3}}\right)\left[-\tilde{h}_{1} \lambda_{1} \lambda_{3}\left(\lambda_{3}-\lambda_{1}\right)+\tilde{h}_{2}\left(\lambda_{3}-\lambda_{1}\right)\left(\lambda_{3}+\lambda_{1}\right)-\tilde{h}_{3}\left(\lambda_{3}-\lambda_{1}\right)\right] \text {, } \\
& \Delta_{3}(s)=\left|\begin{array}{ccc}
1-e^{\lambda_{1}} & 1-e^{\lambda_{2}} & \tilde{h}_{1} \\
\lambda_{1}\left(1-e^{\lambda_{1}}\right) & \lambda_{2}\left(1-e^{\lambda_{2}}\right) & \tilde{h}_{2} \\
\lambda_{1}^{2}\left(1-e^{\lambda_{1}}\right) & \lambda_{2}^{2}\left(1-e^{\lambda_{2}}\right) & \tilde{h}_{3}
\end{array}\right| \\
& =\left(1-e^{\lambda_{2}}\right)\left(1-e^{\lambda_{1}}\right)\left[\tilde{h}_{1} \lambda_{2} \lambda_{1}\left(\lambda_{2}-\lambda_{1}\right)-\tilde{h}_{2}\left(\lambda_{2}-\lambda_{1}\right)\left(\lambda_{2}+\lambda_{1}\right)+\tilde{h}_{3}\left(\lambda_{2}-\lambda_{1}\right)\right],
\end{aligned}
$$

Note that for $s=\alpha+\beta i$ with $\alpha>0$ and $s \neq \frac{4}{27}$, the determinant $\Delta(s) \neq 0^{3}$ since $\Delta(s)=0$ if and only if $s$ is an eigenvalue of the operator

$$
A=-D_{x}^{3}+D_{x}^{2}: L^{2}(0,1) \rightarrow L^{2}(0,1)
$$

with the domain

$$
\mathcal{D}(A)=\left\{v \in H^{s}(0,1) ; v(0)=v(1), v^{\prime}(0)=v^{\prime}(1), v^{\prime \prime}(0)=v^{\prime \prime}(1)\right\}
$$

It thus follows from the Cramer's rule that

$$
\tilde{u}(x, s)=\sum_{j=1}^{3} \frac{\Delta_{j}(s)}{\Delta(s)} e^{\lambda_{j}(s) x} .
$$

which we rewrite as

$$
\tilde{u}(x, s)=\sum_{k=1}^{3} \tilde{u}_{k}(x, s)
$$

with

$$
\tilde{u}_{k}(x, s)=\sum_{j=1}^{3} \frac{R_{k j}(s)}{1-e^{\lambda_{j}(s)}} e^{\lambda_{j}(s) x} \tilde{h}_{k}(s), \quad k=1,2,3
$$

\footnotetext{
${ }^{3}$ If there is no dissipation, $A=-D_{x}^{3}$, then $\Delta(s)$ have infinitely many zeros on the imaginary axis $s=\beta i$.
} 
where

$$
\begin{gathered}
R_{11}(s)=\frac{\lambda_{2} \lambda_{3}}{\left(\lambda_{3}-\lambda_{1}\right)\left(\lambda_{2}-\lambda_{1}\right)}, R_{12}(s)=\frac{\lambda_{1} \lambda_{3}}{\left(\lambda_{1}-\lambda_{2}\right)\left(\lambda_{3}-\lambda_{2}\right)}, R_{13}(s)=\frac{\lambda_{1} \lambda_{2}}{\left(\lambda_{1}-\lambda_{3}\right)\left(\lambda_{2}-\lambda_{3}\right)}, \\
R_{21}(s)=-\frac{\lambda_{3}+\lambda_{2}}{\left(\lambda_{3}-\lambda_{1}\right)\left(\lambda_{2}-\lambda_{1}\right)}, R_{22}(s)=-\frac{\lambda_{3}+\lambda_{1}}{\left(\lambda_{1}-\lambda_{2}\right)\left(\lambda_{3}-\lambda_{2}\right)}, R_{23}(s)=-\frac{\lambda_{2}+\lambda_{1}}{\left(\lambda_{3}-\lambda_{1}\right)\left(\lambda_{3}-\lambda_{2}\right)}, \\
R_{31}(s)=\frac{1}{\left(\lambda_{1}-\lambda_{3}\right)\left(\lambda_{1}-\lambda_{2}\right)}, R_{32}(s)=\frac{1}{\left(\lambda_{2}-\lambda_{1}\right)\left(\lambda_{2}-\lambda_{3}\right)}, R_{33}(s)=\frac{1}{\left(\lambda_{3}-\lambda_{1}\right)\left(\lambda_{3}-\lambda_{2}\right)} .
\end{gathered}
$$

Taking the inverse Laplace transform of $\tilde{u}$ yields the representation

$$
u(x, t)=\sum_{k=1}^{3} u_{k}(x, t)
$$

with

$$
u_{k}(x, t)=\frac{1}{2 \pi i} \int_{r-i \infty}^{r+i \infty} e^{s t} \tilde{u}_{k}(x, s) \mathrm{d} s=\sum_{j=1}^{3} \frac{1}{2 \pi i} \int_{r-i \infty}^{r+i \infty} e^{s t} \frac{R_{k j}(s)}{1-e^{\lambda_{j}(s)}} e^{\lambda_{j}(s) x} \tilde{h}_{k}(s) \mathrm{d} s,
$$

for $k=1,2,3$, where $r>0$ is a fixed constant with $r \neq \frac{4}{27}$. For given $0<r<\frac{4}{27}$, let

$$
\Omega_{r}=\{\alpha+\beta i: 0<\alpha<r,-\infty<\beta<\infty\}
$$

The following four lemmas, which will play important roles in estimating the solutions of (3.1), follow from direct computations.

Lemma 3.1. Let $s=\alpha+i \beta$ with $\alpha \geq 0, \rho^{3}=\sqrt{\alpha^{2}+\beta^{2}}$ and $3 \theta=\tan ^{-1} \frac{\beta}{\alpha}$. Then three solutions of

$$
s+\lambda^{3}-\lambda^{2}=0
$$

have the following asymptotic form as $\rho \rightarrow+\infty$ :

$$
\lambda_{1}=\frac{1}{3}+\rho e^{i(\theta+\pi / 3)}+O\left(\rho^{-1}\right), \quad \lambda_{2}=\frac{1}{3}+\rho e^{i(\theta+\pi)}+O\left(\rho^{-1}\right), \quad \lambda_{3}=\frac{1}{3}+\rho e^{i(\theta+(5 \pi / 3))}+O\left(\rho^{-1}\right) .
$$

Moreover, there exists a constant $\epsilon>0$ such that

$$
\sup _{s \in \Omega_{r}}\left|1-e^{\lambda_{j}(s)}\right|>\epsilon, \quad j=1,2,3 .
$$

For given $x \in(0,1)$

$$
H_{j k}(s):=\frac{R_{k j}(s)}{1-e^{\lambda_{j}(s)}} e^{\lambda_{j}(s) x} \tilde{h}_{k}(s), j, k=1,2,3,
$$


is analytic in $\Omega_{r}$ and continuous on $\overline{\Omega_{r}}$, one may let $r \rightarrow 0$ to obtain,

$$
\begin{aligned}
u_{k j}(x, t) & =\frac{1}{2 \pi i} \int_{r-i \infty}^{r+i \infty} e^{s t} \frac{R_{k j}(s)}{1-e^{\lambda_{j}(s)}} e^{\lambda_{j}(s) x} \tilde{h}_{k}(s) \mathrm{d} s \\
& =\frac{1}{2 \pi i} \int_{-i \infty}^{i \infty} e^{s t} \frac{R_{k j}(s)}{1-e^{\lambda_{j}(s)}} e^{\lambda_{j}(s) x} \tilde{h}_{k}(s) \mathrm{d} s \\
& =\frac{1}{2 \pi i} \int_{0}^{i \infty} e^{s t} \frac{R_{k j}(s)}{1-e^{\lambda_{j}(s)}} e^{\lambda_{j}(s) x} \tilde{h}_{k}(s) \mathrm{d} s+\frac{1}{2 \pi i} \int_{-i \infty}^{0} e^{s t} \frac{R_{k j}(s)}{1-e^{\lambda_{j}(s)}} e^{\lambda_{j}(s) x} \tilde{h}_{k}(s) \mathrm{d} s \\
& =\frac{1}{2 \pi i} \int_{0}^{i \infty} e^{s t} \frac{R_{k j}(s)}{1-e^{\lambda_{j}(s)}} e^{\lambda_{j}(s) x} \tilde{h}_{k}(s) \mathrm{d} s+\frac{1}{2 \pi i} \int_{0}^{i \infty} e^{s t} \frac{R_{k j}(s)}{1-e^{\lambda_{j}(s)}} e^{\lambda_{j}(s) x} \tilde{h}_{k}(s) \mathrm{d} s \\
& =u_{k j}^{+}(x, t)+\frac{u_{k j}^{+}(x, t)}{}
\end{aligned}
$$

where

$$
\begin{aligned}
u_{k j}^{+}(x, t) & =\frac{1}{2 \pi i} \int_{0}^{i \infty} e^{s t} \frac{R_{k j}(s)}{1-e^{\lambda_{j}(s)}} e^{\lambda_{j}(s) x} \tilde{h}_{k}(s) \mathrm{d} s \\
& =\frac{1}{2 \pi} \int_{0}^{\infty} e^{i\left(\rho^{3}+\frac{1}{3} \rho\right) t} \frac{R_{k j}^{+}(\rho)}{1-e^{\lambda_{j}^{+}(\rho)}} e^{\lambda_{j}^{+}(\rho) x}\left(3 \rho^{2}+\frac{1}{3}\right) \tilde{h}_{k}^{+}(\rho) \mathrm{d} \rho
\end{aligned}
$$

with

$$
R_{k j}^{+}(\rho):=R_{k j}\left(i \rho^{3}+\frac{i}{3} \rho\right), \quad \lambda_{j}^{+}(\rho):=\lambda_{j}\left(i \rho^{3}+\frac{i}{3} \rho\right), \quad \tilde{h}_{k}^{+}(\rho):=\tilde{h}_{k}\left(i \rho^{3}+\frac{i}{3} \rho\right)
$$

for $k, j=1,2,3$.

Lemma 3.2. As $\rho \rightarrow+\infty$,

$$
\begin{gathered}
\lambda_{1}^{+}(\rho)=\frac{1}{3}+i \rho+O\left(\rho^{-1}\right), \\
\lambda_{2}^{+}(\rho)=\frac{1}{3}+\sqrt{\frac{3 \rho^{2}}{4}+\frac{1}{3}}-\frac{\rho i}{2}+O\left(\rho^{-1}\right)=\frac{1}{3}+\frac{\sqrt{3}-i}{2} \rho+o(1),
\end{gathered}
$$

and

$$
\left.\lambda_{3}^{+}(\rho)=\frac{1}{3}-\sqrt{\frac{3 \rho^{2}}{4}+\frac{1}{3}}-\frac{\rho i}{2}+O\left(\rho^{-1}\right)\right)=\frac{1}{3}-\frac{\sqrt{3}+i}{2} \rho+o(1) .
$$

Lemma 3.3. As $\rho \rightarrow+\infty$,

$$
\begin{gathered}
R_{11}^{+}(\rho)=\alpha_{1,1}+O\left(\rho^{-1}\right), \\
R_{k j}^{+}(\rho)=\frac{\alpha_{k, j}}{\rho}+O\left(\rho^{-2}\right), \quad k=1,2, j=2,3,
\end{gathered}
$$




$$
R_{k j}^{+}(\rho)=\frac{\alpha_{k, j}}{\rho^{2}}+O\left(\rho^{-3}\right), \quad k=2, j=1, \quad \text { or } k=3, j=1,2,3
$$

where $\alpha_{k, j}$ are nonzero constants for $j, k=1,2,3$. Moreover,

$$
1-e^{\lambda_{1}^{+}(\rho)} \sim 1-e^{\frac{1}{3}+\rho i}, \quad 1-e^{\lambda_{2}^{+}(\rho)} \sim e^{\frac{\sqrt{3}-i}{2} \rho}, \quad 1-e^{\lambda_{3}^{+}(\rho)} \sim 1
$$

as $\rho \rightarrow+\infty$.

Lemma 3.4. For $k=1,2,3, j=1,3$, let

$$
\tilde{h}_{k, j}^{*^{+}}(\rho):=\frac{R_{k j}^{+}(\rho)}{1-e^{\lambda_{j}^{+}(\rho)}} \tilde{h}_{k}^{+}(\rho)
$$

and

$$
\tilde{h}_{k, 2}^{*^{+}}(\rho):=\frac{R_{k 2}^{+}(\rho)}{1-e^{\lambda_{2}^{+}(\rho)}} e^{\lambda_{2}^{+}(\rho)} \tilde{h}_{k}^{+}(\rho) .
$$

Then,

$$
h_{1} \in H_{0}^{\frac{s+1}{3}}\left(\mathbb{R}^{+}\right), h_{2} \in H_{0}^{s}\left(\mathbb{R}^{+}\right), h_{3} \in H_{0}^{\frac{s-1}{3}}\left(\mathbb{R}^{+}\right) \Longrightarrow \tilde{h}_{k, j}^{*} \in H^{\frac{s+1}{3}}(\mathbb{R})
$$

for $k, j=1,2,3$ with

$$
\left\|\tilde{h}_{1, j}^{*^{+}}\right\|_{H^{\frac{s+1}{3}(\mathbb{R})}} \leq C_{s}\left\|h_{1}\right\|_{H^{\frac{s+1}{3}}\left(\mathbb{R}^{+}\right)}, \quad\left\|\tilde{h}_{2, j}^{*^{+}}\right\|_{H^{\frac{s+1}{3}}(\mathbb{R})} \leq C_{s}\left\|h_{2}\right\|_{H^{\frac{s}{3}}\left(\mathbb{R}^{+}\right)}
$$

and

$$
\left\|\tilde{h}_{3, j}^{*+}\right\|_{H^{\frac{s+1}{3}}(\mathbb{R})} \leq C_{s}\left\|h_{3}\right\|_{H^{\frac{s-1}{3}}\left(\mathbb{R}^{+}\right)}
$$

for $j=1,2,3$.

For given $k, j=1,2,3$, let $\mathcal{B}_{k, j}$ be an operator from $H_{0}^{s}\left(\mathbb{R}^{+}\right)$defined as follows: for any $h \in H_{0}^{s}(\mathbb{R})$,

$$
\left[\mathcal{B}_{k, j} h\right](x, t)=\left[U_{k, j} h\right](x, t)+\overline{\left[U_{k, j} h\right](x, t)}
$$

with

$$
\left[U_{k, j} h\right](x, t)=\frac{1}{2 \pi} \int_{0}^{\infty} e^{i\left(\rho^{3}+\frac{1}{3} \rho\right) t} e^{\lambda_{j}^{+}(\rho) x}\left(3 \rho^{2}+1 / 3\right) \frac{R_{k j}^{+}(\rho)}{1-e^{\lambda_{j}^{+}(\rho)}} \tilde{h}^{+}(\rho) \mathrm{d} \rho
$$

for $k=1,2,3, j=1,3$ and

$$
\left[U_{k, 2} h\right](x, t)=\frac{1}{2 \pi} \int_{0}^{\infty} e^{i\left(\rho^{3}+\frac{1}{3} \rho\right) t} e^{-\lambda_{2}^{+}(\rho)(1-x)}\left(3 \rho^{2}+1 / 3\right) \frac{R_{k 2}^{+}(\rho)}{1-e^{\lambda_{2}^{+}(\rho)}} e^{\lambda_{2}^{+}(\rho)} \tilde{h}^{+}(\rho) \mathrm{d} \rho
$$


for $k=1,2,3$. Here

$$
\tilde{h}^{+}(\rho)=\tilde{h}\left(i \rho^{3}+\frac{i}{3} \rho\right)
$$

Proposition 3.5. For given $h_{1}, h_{2}$, and $h_{3}$, let $\vec{h}=\left(h_{1}, h_{2}, h_{3}\right)$. Then the solution of (3.1) may be written in the form

$$
u(x, t)=[\mathcal{B} \vec{h}](x, t):=\sum_{k, j=1}^{3}\left[\mathcal{B}_{k, j} h_{k}\right](x, t)
$$

In particular,

$$
\left[\mathcal{B}_{k, j} h_{k}\right](x, t)=\left[U_{k, j} h_{k}\right](x, t)+\overline{\left[U_{k, j} h_{k}\right](x, t)}
$$

with

$$
\left[U_{k, j} h_{k}\right](x, t)=\frac{1}{2 \pi} \int_{0}^{\infty} e^{i\left(\rho^{3}+\frac{1}{3} \rho\right) t} e^{\lambda_{j}^{+}(\rho) x}\left(3 \rho^{2}+1 / 3\right) \tilde{h}_{k, j}^{*^{+}}(\rho) \mathrm{d} \rho
$$

for $k=1,2,3, j=1,3$ and

$$
\left[U_{k, 2} h_{k}\right](x, t)=\frac{1}{2 \pi} \int_{0}^{\infty} e^{i\left(\rho^{3}+\frac{1}{3} \rho\right) t} e^{-\lambda_{2}^{+}(\rho)(1-x)}\left(3 \rho^{2}+1 / 3\right) \tilde{h}_{k, 2}^{*+}(\rho) \mathrm{d} \rho
$$

for $k=1,2,3$. Here, $\tilde{h}_{k, j}^{*^{+}}(\rho)$ are as defined in Lemma 3.4.

The operator $\mathcal{B}$ is called as the boundary integral operator.

For any $s \in \mathbb{R}$, let

$$
\mathcal{H}^{s}\left(\mathbb{R}^{+}\right):=H_{0}^{\frac{s+1}{3}}\left(\mathbb{R}^{+}\right) \times H_{0}^{\frac{s}{3}}\left(\mathbb{R}^{+}\right) \times H_{0}^{\frac{s-1}{3}}\left(\mathbb{R}^{+}\right) .
$$

Using the same arguments as that in the proof of Theorem 2.10 in [2], one can see that if $\vec{h} \in \mathcal{H}^{0}\left(\mathbb{R}^{+}\right)$, then

$$
u=\mathcal{B} \vec{h} \in C_{b}\left(\mathbb{R}^{+} ; L^{2}(0,1)\right) \cap L^{2}\left(\mathbb{R}^{+} ; H^{1}(0,1)\right)
$$

with

$$
\sup _{0<t<\infty}\|u(\cdot, t)\|_{L^{2}(0,1)}+\|u\|_{L^{2}\left(\mathbb{R}^{+}, H^{1}(0,1)\right)} \leq C\|\vec{h}\|_{\mathcal{H}^{0}\left(\mathbb{R}^{+}\right)}
$$

Moreover, it possesses the sharp Kato smoothing property as described by the following proposition.

Proposition 3.6. For any $\vec{h}=\mathcal{H}^{0}\left(\mathbb{R}^{+}\right)$, the solution $u$ of (3.1) satisfies

$$
\sup _{0<x<1}\left\|u_{x}(x, \cdot)\right\|_{L^{2}\left(\mathbb{R}^{+}\right)} \leq C\|\vec{h}\|_{\mathcal{H}^{0}\left(\mathbb{R}^{+}\right)}
$$


Proof. For any $\vec{h} \in \mathcal{H}^{0}\left(\mathbb{R}^{+}\right)$, the solution $u$ of (3.1) is given by

$$
u(x, t)=[\mathcal{B} \vec{h}](x, t)=\sum_{k, j=1}^{3}\left\{\left[U_{k, j} h_{k}\right](x, t)+\overline{\left[U_{k, j} h_{k}\right](x, t)}\right\} .
$$

We only consider the case of

$$
v(x, t):=\left[U_{1,1} h_{1}\right](x, t)=\frac{1}{2 \pi} \int_{0}^{\infty} e^{i\left(\rho^{3}+\frac{1}{3} \rho\right) t} e^{\lambda_{1}^{+}(\rho) x}\left(3 \rho^{2}+1 / 3\right) \tilde{h}_{1,1}^{*^{+}}(\rho) \mathrm{d} \rho,
$$

the proofs for other cases are similar.

As

$$
\begin{aligned}
v_{x}(x, t) & =\frac{1}{2 \pi} \int_{0}^{\infty} e^{i\left(\rho^{3}+\frac{1}{3} \rho\right) t} e^{\lambda_{1}^{+}(\rho) x} \lambda_{1}^{+}(\rho)\left(3 \rho^{2}+1 / 3\right) \tilde{h}_{1,1}^{*+}(\rho) \mathrm{d} \rho \\
& =\frac{1}{2 \pi} \int_{0}^{\infty} e^{i \eta t} e^{\lambda_{1}^{+}(\mu(\eta)) x} \lambda_{1}^{+}(\mu(\eta)) \tilde{h}_{1,1}^{*^{+}}(\mu(\eta)) \mathrm{d} \eta
\end{aligned}
$$

where $\rho=\mu(\eta)$ is the solution of the equation

$$
\eta=\rho^{3}+\frac{1}{3} \rho
$$

applying Plancherel Theorem with respect to $t$ yields

$$
\left\|v_{x}(x, \cdot)\right\|_{L^{2}\left(\mathbb{R}^{+}\right)}^{2}=c \int_{\mathbb{R}^{+}}\left|e^{\lambda_{1}^{+}(\mu(\eta)) x}\right|^{2}\left|\lambda_{1}^{+}(\mu(\eta))\right|^{2}\left|\tilde{h}_{1,1}^{*+}(\mu(\eta))\right|^{2} \mathrm{~d} \eta .
$$

Recalling Lemma 3.2 and Lemma 3.4, we arrive at

$$
\sup _{0<x<1}\left\|v_{x}(x, \cdot)\right\|_{L^{2}\left(\mathbb{R}^{+}\right)}^{2} \leq c \int_{\mathbb{R}^{+}}\left|\lambda_{1}^{+}(\mu(\eta))\right|^{2}\left|\tilde{h}_{1,1}^{*^{+}}(\mu(\eta))\right|^{2} \mathrm{~d} \eta \leq c\left\|h_{1}\right\|_{H^{\frac{1}{3}}\left(\mathbb{R}^{+}\right)}^{2} .
$$

\subsection{Linear problems}

For the Cauchy problem of the linear KdV-Burgers equation posed on $\mathbb{R}$,

$$
w_{t}+w_{x x x}-w_{x x}=g(x, t), \quad w(x, 0)=\psi(x), \quad x \in \mathbb{R}, t>0,
$$

for any given $s \in \mathbb{R}, T>0, \psi \in H^{s}(\mathbb{R})$ and $g \in L^{1}\left(0, T ; H^{s}(\mathbb{R})\right)$, it admits a unique solution

$$
w \in C\left([0, T] ; H^{s}(\mathbb{R})\right),
$$

which can be written as the form of

$$
w(x, t)=W_{\mathbb{R}}(t) \psi+\int_{0}^{t} W_{\mathbb{R}}(t-\tau) g(\tau) \mathrm{d} \tau,
$$


where $W_{\mathbb{R}}(t)$ is the $C_{0}$-semigroup associated to the Cauchy problem (3.4),

$$
W_{\mathbb{R}}(t) \psi=\int_{\mathbb{R}} e^{i \xi^{3} t-\xi^{2} t} e^{i \xi x} \hat{\psi}(\xi) \mathrm{d} \xi
$$

with $\hat{\psi}$ being the Fourier transform of $\psi$.

The solutions of (3.4) not only possess the Kato smoothing property, i.e., given $s \in \mathbb{R}$,

$$
\psi \in H^{s}(\mathbb{R}), g \in L^{1}\left(\mathbb{R}^{+} ; H^{s}(\mathbb{R})\right) \Longrightarrow w \in C_{b}\left(\mathbb{R}^{+} ; H^{s}(\mathbb{R})\right) \cap L^{2}\left(\mathbb{R}^{+} ; H^{s+1}(\mathbb{R})\right)
$$

and

$$
\psi \in H^{s}(\mathbb{R}), g \in L^{2}\left(\mathbb{R}^{+} ; H^{s-1}(\mathbb{R})\right) \Longrightarrow w \in C_{b}\left(\mathbb{R}^{+} ; H^{s}(\mathbb{R})\right) \cap L^{2}\left(\mathbb{R}^{+} ; H^{s+1}(\mathbb{R})\right),
$$

but also possess the sharp Kato smoothing property as described by the following proposition.

Proposition 3.7. Let $s \in \mathbb{R}$ be given. Then

$$
\psi \in H^{s}(\mathbb{R}), g \in L^{1}\left(\mathbb{R}^{+} ; H^{s}(\mathbb{R})\right) \Longrightarrow \partial_{x}^{s+1} w \in L_{x}^{\infty}\left(\mathbb{R} ; L^{2}\left(\mathbb{R}^{+}\right)\right)
$$

satisfying

$$
\left\|\partial_{x}^{s+1} w\right\|_{L_{x}^{\infty}\left(\mathbb{R} ; L^{2}\left(\mathbb{R}^{+}\right)\right)} \leq C\left(\|\psi\|_{H^{s}(\mathbb{R})}+\|g\|_{L^{1}\left(\mathbb{R}^{+} ; H^{s}(\mathbb{R})\right)}\right) .
$$

Proof. It suffices to consider only the case of $s=0$.

Assume first $g \equiv 0$ and $\psi \in L^{2}(\mathbb{R})$. The solution

$$
w(x, t)=W_{\mathbb{R}}(t) \psi=\int_{\mathbb{R}} e^{i \xi^{3} t-\xi^{2} t} e^{i x \xi} \hat{\psi}(\xi) \mathrm{d} \xi
$$

and

$$
w_{x}(x, t)=\int_{\mathbb{R}} e^{i \xi^{3} t-\xi^{2} t} e^{i x \xi} i \xi \hat{\psi}(\xi) \mathrm{d} \xi=\int_{\mathbb{R}} e^{i t\left(\eta+i \eta^{\frac{2}{3}}\right)} e^{i x \eta^{\frac{1}{3}}}\left(i \eta^{\frac{1}{3}}\right) \hat{\psi}\left(\eta^{\frac{1}{3}}\right) \frac{1}{3} \eta^{-\frac{2}{3}} \mathrm{~d} \eta .
$$

Using Lemma 2.6 in [4] (a revised version of the Plancherel theorem) with respect to $t$ variable,

$$
\sup _{x \in \mathbb{R}}\left\|w_{x}(x, \cdot)\right\|_{L_{t}^{2}\left(\mathbb{R}^{+}\right)}^{2} \leq C \int_{\mathbb{R}}|\eta|^{-\frac{2}{3}}\left|\hat{\psi}\left(\eta^{\frac{1}{3}}\right)\right|^{2} \mathrm{~d} \eta \leq C\|\psi\|_{L^{2}(\mathbb{R})}^{2} .
$$

Thus

$$
\left\|\partial_{x} W_{\mathbb{R}}(t) \psi\right\|_{L_{x}^{\infty}\left(\mathbb{R} ; L_{t}^{2}\left(\mathbb{R}^{+}\right)\right)} \leq C\|\psi\|_{L^{2}(\mathbb{R})} .
$$

If $\psi=0, g \in L^{1}\left(\mathbb{R}^{+} ; L^{2}(\mathbb{R})\right)$, then

$$
w(x, t)=\int_{0}^{t} W_{\mathbb{R}}(t-\tau) g(\tau) \mathrm{d} \tau
$$


and

$$
\left\|w_{x}\right\|_{L_{x}^{\infty}\left(\mathbb{R} ; L_{t}^{2}\left(\mathbb{R}^{+}\right)\right)} \leq \int_{0}^{\infty}\left\|\partial_{x} W_{\mathbb{R}}(t-\tau) g(\tau)\right\|_{L_{x}^{\infty}\left(\mathbb{R} ; L_{t}^{2}((\tau,+\infty))\right.} \mathrm{d} \tau \leq C \int_{0}^{\infty}\|g(\tau)\|_{L^{2}(\mathbb{R})} \mathrm{d} \tau
$$

Consider the Cauchy problem of the linear KdV-Burgers equation posed on the interval $(0,1)$ with periodic boundary conditions

$$
\left\{\begin{array}{l}
u_{t}+u_{x x x}-u_{x x}=f(x, t), u(x, 0)=\phi(x), x \in(0,1), t>0 \\
u(0, t)=u(1, t), u_{x}(0, t)=u_{x}(1, t), u_{x x}(0, t)=u_{x x}(1, t) .
\end{array}\right.
$$

Let

$$
\phi_{n}(x)=e^{i 2 n \pi x}, \quad n=0, \pm 1, \pm 2, \cdots
$$

Then $\left\{\phi_{n}\right\}_{-\infty}^{\infty}$ forms an orthonormal basis in the space $L^{2}(0,1)$. We may define the Sobolev space $H_{p}^{s}:=H_{p}^{s}(0,1)$ of order $s(s \in \mathbb{R})$ as the space of all real periodic functions of period 1

$$
v(x)=\sum_{n=-\infty}^{\infty} v_{n} \phi_{n}(x)
$$

such that

$$
\left\{\sum_{n=-\infty}^{\infty}\left|v_{n}\right|^{2}(1+|n|)^{2 s}\right\}^{\frac{1}{2}}<+\infty
$$

The left hand side of (3.6) is a Hilbert norm for $H_{p}^{s}$; we denote it by $\|v\|_{s}$.

$$
W_{\mathbb{T}}(t) \phi=\sum_{k=-\infty}^{\infty} e^{i k^{3} t-k^{2} t} \hat{\phi}(k) \phi_{k}(x) .
$$

For any $s \in \mathbb{R}, v \in H_{p}^{s}$ with

$$
v(x)=\sum_{k=-\infty}^{\infty} v_{k} \phi_{k}(x)
$$

we define the operator $D_{x}^{s}$ by

$$
D_{x}^{s} v(x)=\sum_{k=-\infty}^{\infty}\left(1+k^{2}\right)^{\frac{s}{2}} v_{k} \phi_{k}(x) .
$$

Thus

$$
\|v\|_{s}=\left\|D_{x}^{s} v\right\|_{L^{2}(0,1)}
$$


Solutions of (3.5) do possess the Kato smoothing property as described by the following proposition.

Given $s \in \mathbb{R}$, for any $\phi \in H_{p}^{s}$ and $f \in L^{1}\left(\mathbb{R}^{+} ; H_{p}^{s}\right)$, (3.5) admits a unique solution

$$
u \in C_{b}\left(\mathbb{R}^{+} ; H_{p}^{s}\right)
$$

which can be written in the form

$$
u(x, t)=W_{\mathbb{T}}(t) \phi+\int_{0}^{t} W_{\mathbb{T}}(t-\tau) f(\tau) \mathrm{d} \tau,
$$

where $W_{\mathbb{T}}(t)$ is the $C_{0}$-semigroup associated with the Cauchy problem (3.5). Solutions of (3.5) possess the Kato smoothing property:

$$
\phi \in H_{p}^{s}, \quad f \in L^{1}\left(\mathbb{R}^{+} ; H_{p}^{s}\right) \Longrightarrow u \in L^{2}\left(\mathbb{R}^{+} ; H_{p}^{s+1}\right)
$$

with

$$
\|u\|_{C_{b}\left(\mathbb{R}^{+} ; H_{p}^{s}\right)}+\|u\|_{L^{2}\left(\mathbb{R}^{+} ; H_{p}^{s+1}\right)} \leq C\left(\|\phi\|_{s}+\|f\|_{L^{1}\left(\mathbb{R}^{+} ; H_{p}^{s}\right)}\right)
$$

and

$$
\phi \in H_{p}^{s}, f \in L^{2}\left(\mathbb{R}^{+} ; H_{p}^{s-1}\right) \Longrightarrow u \in L^{2}\left(\mathbb{R}^{+} ; H_{p}^{s+1}\right)
$$

with

$$
\|u\|_{C_{b}\left(\mathbb{R}^{+} ; H_{p}^{s}\right)}+\|u\|_{L^{2}\left(\mathbb{R}^{+} ; H_{p}^{s+1}\right)} \leq C\left(\|\phi\|_{s}+\|f\|_{L^{2}\left(\mathbb{R}^{+} ; H_{p}^{s-1}\right)}\right),
$$

where the constant $C$ depends only on $s$.

The next proposition shows that the solutions of (3.5) also possess the sharp Kato smoothing property.

Proposition 3.8. Let $s \in \mathbb{R}$ be given. For any $\phi \in H_{p}^{s}$ and $f \in L^{1}\left(\mathbb{R}^{+} ; H_{p}^{s}\right)$, the corresponding solution $u$ of (3.5) in the space $C_{b}\left(\mathbb{R}^{+} ; H_{p}^{s}\right)$ satisfies

$$
\sup _{0<x<1}\left\|\partial_{x}^{1+s} u(x, \cdot)\right\|_{L^{2}\left(\mathbb{R}^{+}\right)} \leq C\left(\|\phi\|_{s}+\|f\|_{L^{1}\left(\mathbb{R}^{+} ; H_{p}^{s}\right)}\right) .
$$

Proof. It suffices to consider the case $s=0$. Using the boundary integral operators introduced in Section 3.1, the solutions of (3.5) can be represented by the solutions of (3.4) in the following way. Let

For $\phi \in L^{2}(0,1)$, let $\phi^{*}$ be the zero extension from $(0,1)$ to $\mathbb{R}$. Then $\phi^{*} \in L^{2}(\mathbb{R})$ with $\left\|\phi^{*}\right\|_{L^{2}(\mathbb{R})}=\|\phi\|_{L^{2}(0,1)}$.

$$
\begin{gathered}
w(x, t)=W_{\mathbb{R}}(t) \phi^{*}, \\
h_{1}(t)=w(0, t)-w(1, t), h_{2}(t)=w_{x}(0, t)-w_{x}(1, t), h_{3}(t)=w_{x x}(0, t)-w_{x x}(1, t) .
\end{gathered}
$$

By Proposition 3.7, $w \in C_{b}\left(\mathbb{R}^{+} ; L^{2}(\mathbb{R})\right) \cap L^{2}\left(\mathbb{R}^{+} ; H^{1}(\mathbb{R})\right)$ and

$$
h_{1} \in H^{\frac{1}{3}}\left(\mathbb{R}^{+}\right), \quad h_{2} \in L^{2}\left(\mathbb{R}^{+}\right), \quad h_{3} \in H^{-\frac{1}{3}}\left(\mathbb{R}^{+}\right)
$$


with

$$
\|\vec{h}\|_{\mathcal{H}^{0}} \leq C\left\|\phi^{*}\right\|_{L^{2}(\mathbb{R})}
$$

When restricted on $(0,1) \times \mathbb{R}^{+}$,

$$
W_{\mathbb{T}}(t) \phi=W_{\mathbb{R}}(t) \phi^{*}-[\mathcal{B} \vec{h}](x, t) .
$$

Consequently the solution $u$ of $(3.5)$ with $f \equiv 0$ can be written as

$$
u(x, t)=W_{\mathbb{T}}(t) \phi=W_{\mathbb{R}}(t) \phi^{*}-[\mathcal{B} \vec{h}](x, t) .
$$

By Proposition 3.6 and Proposition 3.7,

$$
\sup _{0<x<1}\left\|\partial_{x}\left[W_{\mathbb{T}}(t) \phi\right](x, \cdot)\right\|_{L^{2}\left(\mathbb{R}^{+}\right)} \leq C\|\phi\|_{L^{2}(0,1)} .
$$

For $\phi \equiv 0$ and $f \in L^{1}\left(\mathbb{R}^{+} ; L^{2}(0,1)\right)$, the solution $u$ of $(3.5)$ is given by

$$
u(x, t)=\int_{0}^{t} W_{\mathbb{T}}(t-\tau) f(\tau) \mathrm{d} \tau=\int_{0}^{\infty} \chi_{(0, t)}(\tau) W_{\mathbb{T}}(t-\tau) f(\tau) \mathrm{d} \tau,
$$

where $\chi_{(0, t)}(\tau)$ is the characteristic function on the interval $(0, t)$. Thus

$$
\sup _{0<x<1}\left\|u_{x}(x, \cdot)\right\|_{L^{2}\left(\mathbb{R}^{+}\right)} \leq \int_{0}^{\infty}\left\|\partial_{x} W_{\mathbb{T}}(t-\tau) f(\tau)\right\|_{L_{x}^{\infty}\left(0,1 ; L^{2}(\tau, \infty)\right)} \mathrm{d} \tau \leq C \int_{0}^{\infty}\|f(\tau)\|_{L^{2}(0,1)} \mathrm{d} \tau .
$$

\subsection{Nonlinear problems}

Consider the Cauchy problem of the KdV-Burgers equation posed on $(0,1)$ with periodic boundary conditions,

$$
\left\{\begin{array}{l}
u_{t}+u u_{x}+u_{x x x}-u_{x x}=0, u(x, 0)=\phi(x), x \in(0,1), t>0 \\
u(0, t)=u(1, t), u_{x}(0, t)=u_{x}(1, t), u_{x x}(0, t)=u_{x x}(1, t)
\end{array}\right.
$$

which is well-known to be globally well-posed in the space $H_{p}^{s}$ for $s \geq 0$ :

Let $s \geq 0$ and $T>0$ be given. For any $\phi \in H_{p}^{s}$, (3.7) admits a unique mild solution

$$
u \in C\left([0, T] ; H_{p}^{s}\right)
$$

which possesses the Kato smoothing property:

$$
\phi \in H_{p}^{s} \Longrightarrow u \in L^{2}\left(0, T ; H_{p}^{s+1}\right) .
$$

It also possesses the sharp Kato smoothing property. 
Proposition 3.9. Let $s \geq 0$ and $T>0$ be given. For any $\phi \in H_{p}^{s}$, the corresponding solution $u$ of (3.7) belongs to the space $C\left([0, T] ; H_{p}^{s}\right) \cap L^{2}\left(0, T ; H_{p}^{s+1}\right)$ and satisfies

$$
\sup _{0<x<1}\left\|\partial_{x}^{s+1} u(x, \cdot)\right\|_{L^{2}(0, T)} \leq \alpha_{s, T}\left(\|\phi\|_{s}\right)
$$

where $\alpha_{s, T}: \mathbb{R}^{+} \rightarrow \mathbb{R}^{+}$is a nondecreasing continuous function.

Proof. The same proof as that of Proposition 2.2, shows that, given $s \geq 0$ and $T>0$, for any $\phi \in H_{p}^{s},(3.7)$ admits a unique solution

$$
u \in Y_{s, T}=C\left([0, T] ; H_{p}^{s}\right) \cap L^{2}\left(0, T ; H_{p}^{s+1}\right)
$$

with

$$
\|u\|_{Y_{s, T}} \leq \beta_{T}(\|\phi\|)\|\phi\|_{s}
$$

where $\beta_{T}: \mathbb{R}^{+} \rightarrow \mathbb{R}^{+}$is a nondecreasing continuous function. Since

$$
\begin{gathered}
u_{t}+u_{x x x}-u_{x x}=f:=-u u_{x}, \quad u(x, 0)=\phi(x), \\
u(x, t)=W_{\mathbb{T}}(t) \phi-\int_{0}^{t} W_{\mathbb{T}}(t-\tau) f(\tau) \mathrm{d} \tau .
\end{gathered}
$$

Applying Proposition 3.8 yields that

$$
\begin{aligned}
\sup _{0<x<1}\left\|\partial_{x}^{1+s} u(x, \cdot)\right\|_{L^{2}(0, T)} & \leq\left\|\partial_{x}^{s+1} W_{\mathbb{T}}(t) \phi\right\|_{L_{x}^{\infty}\left(0,1 ; L^{2}(0, T)\right)}+\left\|\partial_{x}^{s+1} \int_{0}^{t} W_{\mathbb{T}}(t-\tau) f(\tau) \mathrm{d} \tau\right\|_{L_{x}^{\infty}\left(0,1 ; L^{2}(0, T)\right)} \\
& \leq C\left(\|\phi\|_{s}+\|f\|_{L^{1}\left(0, T ; H_{p}^{s}\right)}\right) \\
& \leq C\left(\|\phi\|_{s}+\|u\|_{Y_{s, T}}^{2}\right) \\
& \leq \alpha_{s, T}\left(\|\phi\|_{s}\right) .
\end{aligned}
$$

\section{REFERENCES}

[1] J.L. Bona, S.-M. Sun and B.-Y. Zhang, A nonhomogeneous boundary-value problem for the Korteweg-de Vries equation in a quarter plane. Trans. Am. Math. Soc. 354 (2002) 427-490.

[2] J.L. Bona, S.M. Sun and B.-Y. Zhang, A nonhomogeneous boundary-value problem for the Korteweg-de Vries Equation on a finite domain. Commun. Partial Differ. Equ. 28 (2003) 1391-1436.

[3] J.L. Bona, S.M. Sun and B.-Y. Zhang, Boundary smoothing properties of the Korteweg-de Vries equation in a quarter plane and applications. Dyn. Partial Differ. Equ. 3 (2006) 1-69.

[4] J.L. Bona, S.M. Sun and B.-Y. Zhang, Nonhomogeneous problems for the Korteweg-de Vries and the Korteweg-de Vries-Burgers equations in a quarter plane. Ann. Inst. Henri Poincaré Anal. Non Linéaire 25 (2008) 1145-1185.

[5] J. Bourgain, Fourier transform restriction phenomena for certain lattice subsets and applications to nonlinear evolution equations, part I: Shrödinger equations. Geom. Funct. Anal. 3 (1993) 107-156.

[6] J. Bourgain, Fourier transform restriction phenomena for certain lattice subsets and applications to nonlinear evolution equations, part II: the KdV-equation. Geom. Funct. Anal. 3 (1993) 209-262.

[7] E. Cerpa, I. Rivas and B.-Y. Zhang, Boundary controllability of the Korteweg-de Vries equation on a bounded domain. SIAM J. Control Optim. 51 (2013) 2976-3010.

[8] M. Christ, J. Colliander and T. Tao, Asymptotics, frequency modulation, and low regularity ill-posedness for canonical defocusing equations. Am. J. Math. 125 (2003) 1235-1293.

[9] J. Colliander, M. Keel, G. Staffilani, H. Takaoka and T. Tao, Sharp global well-posedness results for periodic and non-periodic KdV and modified KdV on R and T. J. Am. Math. Soc. 16 (2003) 705-749. 
[10] J. Colliander and C. Kenig, The generalized Korteweg-de Vries equation on the half line. Commun. Partial Differ. Equ. 27 (2002) 2187-2266.

[11] P. Constantin and J.-C. Saut, Local smoothing properties of dispersive equations. J. Am. Math. Soc. 1 (1988) $413-446$.

[12] C. Flores and D. Smith, Control and stabilization of the periodic fifth order Korteweg-de Vries equation. ESAIM: COCV 25 (2019) 1-38.

[13] J. Holmer, The initial-boundary value problem for the Korteweg-de Vries equation. Commun. Partial Differ. Equ. 31 (2006) $1151-1190$.

[14] J. Holmer, The initial-boundary-value problem for the 1D nonlinear Schrödinger equation on the half-line. Differ. Integr. Equ. 18 (2005) 647-668.

[15] C. Jia and B.-Y. Zhang, Boundary stabilization of the Korteweg-de Vries equation and the Kortweg-de Vries-Burgers equation. Acta Appl. Math. 118 (2012) 25-47.

[16] T. Kato, On the Korteweg-de Vries equation. Manuscr. Math. 28 (1979) 89-99.

[17] T. Kato, On the Cauchy problem for the (generalized) Korteweg-de Vries equations. Adv. Math. Suppl. Stud. 8 (1983) 93-128.

[18] C. Kenig, G. Ponce and L. Vega, On the (generalized) Korteweg-de Vries equation. Duke Math. J. 59 (1989) 585-610.

[19] C. Kenig, G. Ponce and L. Vega, Oscillatory integrals and regularity of dispersive equations. Indiana Univ. Math. J. 40 (1991) 33-69.

[20] C. Kenig, G. Ponce and L. Vega, Well-posedness of the initial value problem for the Korteweg-de Vries equation. J. Am. Math. Soc. 4 (1991) 323-347.

[21] C. Kenig, G. Ponce and L. Vega, Well-Posedness and scattering results for the generalized Korteweg-de Vries equation via the contraction principle. Commun. Pure Appl. Math. 46 (1993) 527-620

[22] C. Kenig, G. Ponce and L. Vega, A Bilinear estimate with applications to the KdV equation. J. Am. Math. Soc. 9 (1996) 573-603.

[23] F. Linares and L. Rosier, Control and stabilization of the Benjamin-Ono equation on a periodic domain. Trans. Am. Math. Soc. 367 (2015) 4595-4626.

[24] A. Pazy, Semigroups of Linear Operators and Applications to Partial Differential Equations. In Vol. 44 of Applied Mathematical Sciences. Springer-Verlag, New York-Berlin-Heidelberg-Tokyo (1983).

[25] L. Rosier and B.-Y. Zhang, Control and stabilization of the Korteweg-de Vries equation: recent progresses. J. Syst. Sci. Complex. 22 (2009) 647-682.

[26] D.L. Russell and B.-Y. Zhang, Smoothing and decay properties of solutions of the Korteweg-de Vries equation on a periodic domain with point dissipation. J. Math. Anal. Appl. 190 (1995) 449-488.

[27] P. Sjölin, Regularity of solutions to the Schrödinger equation. Duke Math. J. 55 (1987) 699-715.

[28] S.-M. Sun, E. Trélat, B.-Y. Zhang and N. Zhong, On sharpness of the local Kato-smoothing property for dispersive wave equations. Proc. Am. Math. Soc. 145 (2017) 653-664.

[29] L. Tartar, Interpolation non linèaire et régularité. J. Funct. Anal. 9 (1972) 469-489. 